\title{
Retention of Urine after radical Hysterectomy for Cervical Cancer
}

\author{
Jyoti Rana $^{1}$, Shi Hui Rong ${ }^{2}$, Suersh Mehata ${ }^{3}$
}

\begin{abstract}
A total of 90 patients with cervical cancer, who underwent radical hysterectomy were included in this study. 25 patients, i.e. $27.8 \%$ were menopausal and 11 patients, i.e. $12.2 \%$ had blood loss more than $500 \mathrm{ml}$. The patients with retention of urine after radical hysterectomy were compared in relation with loss of blood volume intra- operatively, parity, and menopause; as the possible causes of retention of urine. The overall frequency of retention of urine among the patients who underwent radical hysterectomy for the cervical cancer was $42.2 \%$. The statistical analysis showed that the relation of urine after radical hysterectomy for cervical cancer with loss of blood volume, parity and menopause was found to be non-significant.
\end{abstract}

Key words: Cervical cancer, Radical hysterectomy, retention of urine, CISC.

\section{Introduction:}

Retention of urine is a common postoperative problem associated with risk of over distention and permanent detrusor damage [1]. Urinary retention is the most common complication following radical surgery for cancer of the uterus [2] and appears to be voiding dysfunction, with the incidence of $8 \%$ to $80 \%$ [3]. Impaired bladder sensation, and residual urine were significantly more common among the patients who had more radical surgery (resection parametrial tissue more than $2 \mathrm{~cm}$ ), as were reduced flow rates and abnormal bladder compliance [4]. The cause of lower urinary tract dysfunction may be mainly from the injury of pelvic nerve that results in a contractile detrussor and low compliance bladder. Several anatomical and clinical studies have shown that the disturbance results from disruption of pelvic autonomic nerve during the dissection of the parametrium [5]. Several authors, mainly from Japan and European countries, have proposed many nerve-sparing surgical approaches to avoid pelvic nerve disruption during radical hysterectomy $[6,7,8]$. In order to maintain bladder function, those nerves (hypogastric, pelvic splanchnic, vesical branches, pelvic plexus) networks should be preserved intact as much as possible unless these attempts sacrifice the therapeutic role of the surgery. Various attempts have been made to preserve urinary function, including recently proposed autonomic nerve-sparing radical hysterectomy techniques $[9,10]$. A detailed anatomic study of the pelvic autonomic nerves was conducted by one of the authors and his colleagues, providing the clues on the development of a theoretical approach for preserving the autonomic nerves when performing a radical hysterectomy. In this preliminary report, a technique for systemic autonomic nerve preservation was described according to anatomical consideration [21]. In a few patients impaired voiding may be asymptomatic, but the majority present with frequent voiding, poor flow, intermittent stream, incomplete emptying, straining to void and hesitancy. In general, it is agreed that patients experienced voiding after a radical hysterectomy. However, the incidence of lower urinary tract dysfunction after a radical hysterectomy has been reported inconclusively. Up to one and half of radical hysterectomy patients experience at least one

\footnotetext{
${ }^{1}$ 1Department of Gynecology and Obstetrics,First Affiliated Hospital of Zhengzhou University, Henan, 450052

P.R. China

2 1Department of Gynecology and Obstetrics,First Affiliated Hospital of Zhengzhou University, Henan, 450052 P.R. China

${ }^{3}$ Department of Epidemiology and biostatistics, College of Pubic Health, Zhnegzhou Univeristy, Henan, 450052 P.R. China
} 
lower urinary tract symptom that develops after surgery over variable periods of time [11, 12]. Nevertheless, it is difficult to determine from published results how much morbidity it caused by these functional disorders. The difficulty arises because this aspect of the problem has not been specifically studied. Evaluation has been difficult because of different instrumentation, diverse follow-up intervals, and limited cases. A retrospective study showed lower urinary tract function urodynamically after a radical hysterectomy and found that the incidence of postoperative bladder dysfunction has been reported between $70 \%$ to $85 \%$ of cases $[13,14]$ and prospectively studied the urinary functions with patients' own control. According to them, the genuine bladder dysfunction developed de novo or worsened after radical hysterectomy may be a direct result of the wide resection of the vagina with its supporting structures. Resection of the upper one-third of the vagina, as well as the base of the broad and uterosacral ligaments also may damage the pelvic and pudendal nerves, resulting in impaired urethral sphincter mechanism. [15, 16, 17, 18]. Several surgical techniques have been constructed in an attempt to preserve the autonomic nerve supply of the pelvis without compromising the radicality of the procedure in order to preserve the bladder function [19]. Prevention or early recognition of urinary retention may avoid long-term voiding difficulty. Difficulty in resumption of spontaneous voiding occurs in over $45 \%$ of patients after radical pelvic surgery for gynecological malignancy [22, 23].

\section{Methods and Materials}

The study was done in the department of gynecology and obstetrics in the first affiliated hospital of Zhengzhou University, Henan, and P.R. of China. A total of ninety patients, who underwent radical hysterectomy for the cervical cancer from IA to IIB stages, during the year of 2003 to 2006 were included in this study. The patients were categorized according to the FIGO classification for uterine cervical cancer. This include: two patients at stage IA, fourtytwo patients at stage IB, twenty-four patients at stage IIA, nineteen patients at stage IIB, one patient at stage IA-IIA, one patient at stage IB-IIA and one at stage IIA-IIB. The most patients who underwent radical hysterectomy for cervical cancer were of stage IB, there were around fourty-two patients. Eighty-one of the ninety (90\%) patients had squamous cell carcinoma and nine of ninety (10\%) had adenocarcinoma, respectively. The operative procedures provided for the patients with cervical cancer were radical hysterectomy with pelvic lymphadenectomy with bilateral salpingoophorectomy (55-patients), with unilateral ovary (2-patients) and bilateral ovaries (33-patients) transposition to an extrapelvic site. Preoperatively all the patients with uterine cervical carcinoma who underwent radical hysterectomy had no complains of lower urinary tract dysfunction, especially retention of urine. So, these patients didn't undergo urodynamic tests and also the time and amount of the last preoperative evacuation of the bladder, the duration of the operation and fluid management were recorded adequately. The patients with retention of urine were diagnosed with the help of measuring residual urine volume postoperatively. These patients were compared with intraoperative loss of blood volume, menopause and parity of the patients as the possible causes of the retention of urine after radical hysterectomy for the cervical carcinoma.

Statistical analyses were done with the help of using SPSS 12.0 software, Chi-square test and calculating the $\mathrm{P}$ value. The statistical significance level was set at $\mathrm{P}<0.05$.

\section{Results}

The frequency of the patients in relation to the age group was found to be 31 to 40 years; there were 29 patients with (32.2\%) cervical carcinoma, who underwent radical hysterectomy within this age group. 
Frequency of the patients of cervical cancer in relation to FIGO stage showed that there were more patients in the stage IB, 42 patients $(46.6 \%)$ of total, who underwent radical hysterectomy for the cervical carcinoma. (Table 1)

Table 1, FIGO staging of the subjects among cervical cancer

Stage No. Of patient \%

I A 22.2 ,

IB 4246.6

IIA 2426.6

IIB 1921.1

IA-IIA 11.1

IB-IIA 11.1

IIA-IIB 11.1

Total 90100

Frequency of distribution of retention of urine among the subjects with cervical cancer, who underwent radical hysterectomy, showed 38 patients (42.2\%) suffered from the postoperative retention of urine after the operation.

Frequency of distribution of blood loss among the patients with cervical cancer showed that only 11 patients. $(12.2 \%)$ had more than $500 \mathrm{ml}$ of intraoperative blood loss.

The frequency of distribution of parity among the patients with cervical cancer, who underwent radical hysterectomy, showed that the 27 patients $(30 \%)$ with cervical carcinoma had para four and the least one had para one.

The frequency of distribution of menopause among the patients with cervical cancer, who underwent radical hysterectomy, showed that 65 patients were none menopause constituting $72.2 \%$ and 25 patients were at menopause constituting $27.8 \%$. (Table 6)

Comparing the retention of urine with intraoperative blood loss, as a possible cause with the help of cross-tabulation method showed that, $\mathrm{x} 2=0.780$ and the $\mathrm{P}=0.517$, which is not significant. It means the retention of urine was not due to the intraoperative loss of blood volume (Table 7).

Comparing the retention of urine with menopause of the patients with cervical carcinoma, who underwent radical hysterectomy showed that, $\mathrm{x} 2=0.549$ and $\mathrm{P}=0.468$, which is not significant. It means that the retention of urine of the patients with cervical carcinoma was not associated with menopause of the patients (Table 8).

Again, comparing retention of urine with the parity of the patients, who underwent radical hysterectomy for cervical carcinoma, indicated that there was no association of retention of urine with parity of the patients; $(\mathrm{x} 2=2.428$, and $\mathrm{P}=0.231)$. (Table 9).

The statistical analysis of the patients who were suffered from the retention of urine after radical hysterectomy, was most significantly or might be due to the extent of the surgery, i e due to radical hysterectomy for cervical carcinoma, not due to the intraoperative loss of blood volume, menopause and parity of the patients. 


\section{Discussion}

Bladder dysfunction, typically the urination difficulty in patients, who have undergone radical hysterectomy, causes deterioration in the patients' quality of life due to physical and mental stress. Many gynecologic oncologists have become knowledgeable about the anatomic distribution of the nerves controlling the bladder function and recently, a great deal of interest has been shown in autonomic nerve sparing surgical techniques. Postoperative bladder dysfunction varies with the size of the tumor, the extent of the dissection during the procedure and the interval of follow-up after the surgery. The pathophysiologic mechanisms of the lower urinary tract's functional changes after a radical hysterectomy are a decrease in the musculo-elastic properties of the bladder wall caused by surgical injury and partial damage to the autonomic innervation of the bladder. A study revealed that the degree of postoperative voiding dysfunction depends on the amount of resected vaginal, paravaginal, and the parametrial tissue[12]. In this study, among the patients with cervical carcinoma, who underwent radical hysterectomy in the first affiliated hospital of Zhengzhou University found that $47.36 \%$ of the women with the stage IB cervical carcinoma had residual urine volume more than $100 \mathrm{ml}$ that persisted for more than thirty-eight days after the surgery even with the appropriate bladder care. Altogether $42.2 \%$ of the patients with cervical carcinoma suffered from the postoperative retention of urine. This statistical value is strongly supported by the different literatures. Moreover, this value is less than the value that was reported by Ralph and his colleagues, which was $67 \%$ of patients had impairment or absence of bladder sensation after radical hysterectomy.

Urologic injury is often occurring as a result of radical hysterectomy. Radical hysterectomy requires resection of parametrium and the upper aspect of the vagina. The extent of the lower urinary tract dysfunction after radical hysterectomy for cervical cancer is associated with the radicality of the operation. The data suggest that the most damaging step for bladder dysfunction was vaginal and paravaginal tissue resection, which may be explained by the anatomy of the parasympathetic fibers, arising from the S2-S4 nerve roots to form the pelvic nerves. These nerves enter the hypogastric sheath, and shortly join with the hypogastric (sympathetic) nerves to form the pelvic plexus very close to the antero-lateral aspect of the lower rectum near the anorectal junction. The pelvic plexus give rise to postganglionic fibres, which lie, as a flat meshed band, on the lateral wall of the upper third of the vagina. These fibres reach the bladder through the deep layer of the cervico-vesical and the vagino-vesical ligaments. When radical hysterectomy is performed, the sacro-uterine ligament is entirely interrupted. The recto-vaginal ligament is also interrupted according to the vaginal resection, which in some patients with locally advanced disease may go beyond the middle third.

Doing these steps the pelvic nerves can be damaged, when the caudal part of the recto-uterine ligament and recto-vaginal ligaments are also cut. Therefore, the interruption of the nerve fibers posteriorly is related to the caudal depth of section of the sacro-uterine and the rectovaginal ligaments, and not to where the line of section of these ligaments in between the uterus and the pelvic wall. Moreover, the part of the pelvic plexus can be removed with the paravaginal tissue surrounding the upper third of the vagina. Again, laterally to the ureter, the cardinal ligament can be resected. When the cardinal ligament is resected medial to the ureter, most of the pelvic plexus is preserved, whereas when it is resected laterally, most of the pelvic plexus is damaged and the connection between the cardinal ligament and the deep layer of cervico-vesical ligament are interrupted. According to the findings of Sislow and Mayo, a prevalence of the sympathetic innervetion due to a complete interruption of parasympathetic fibers and partially likely etiology. Due to the imbalance between the parasympathetic and sympathetic innervations the damage of the nerves leads to change the storage function of the bladder causing the retention of urine. So, nowadays, the systemic 
autonomic nerve preservation techniques are developing, which preserve the pelvic plexus and the branches innervating the bladder with the help of "Nerve-Sparing Radical Hysterectomy". According to the study, the technique of preservation of the pelvic autonomic nerves improved the long-term prognosis of bladder dysfunction resulting normal urinary function within 1-year.

In conclusions, long-term urinary tract dysfunction is a frequent complication of radical hysterectomy. Nevertheless, the clinical significance of bladder dysfunction can be greatly reduced by appropriate postoperative bladder care. A careful urologic follow-up, including urodynamic assessment and kidney ultrasound is useful to select the patients at risk for severe urinary complications.

\section{REFERENCES:}

1. Tammela T, Konturri M, Lukkarianen O, Tammela T, Konturri M, et al. Postoperative urinary retention - why the patient can not void. Scand J Urol Nephrol 1995:175:75-77.

2. Vervest HAM, Barents JW, Haspels AA, Deruyne FMJ. Radical hysterectomy and the function of the lower urinary tract. Acta Obstect Gynecol Scand 1989:68:331-40.

3. Zullo MA, Manci N, Angioli R, Muzii L, Panici PB. Vesical dysfunction after radical hysterectomy for cervical cancer: a critical review. Crit Rev Oncol hematol 2003; 48:28793.

4. Tamussino K, Winter R, Lang PFJ. The cardinal ligament: surgical anatomy and resection. CME J Gynecol Onclo 1997; 6:265-71.

5. Mass CP, Kenter GG, Trimbos JB, Deruiter MC. Anatomical basis for nerve-sparing radical hysterectomy: immunological study of the pelvic autonomic nerves. Acta Obstet Gynecol Scand 2005;84:868-74.

6. Raspagliesi F, Ditto A, Fontanell R et al. Nerve-sparing radical hysterectomy: a surgical technique for preserving the autonomic hypogastric nerve, Gnecol Oncol 2004; 93:30414.

7. Kato T, Murakani G, Yabuki Y. A new perspective on nerve-sparing radical hysterectomy: nerve topography and over-preservation of the cardinal ligament. J Clin Oncol 2003; 33:589-91.

8. Sakuragi N, Todo Y, Kudo M, Yamamoto R, sato T. A systematic nerve-sparing radical hysterectomy technique in invasive cervical cancer for preserving postsurgical bladder function, Int J Gynecol Cancer 2005; 15:389-97.

9. Kuwabara Y, Suzuki M, Hashimoto M, Furugen Y, Yoshida K, Mitsuhashi N. New method to prevent bladder dysfunction after radical hysterectomy for uterine cervical cancer. J Obstet Gynecol Res 2002; 26:1-8.

10. Possover M, Stober S, Plaul K, Schneider A. Identification and preservation of the motoric innervation of bladder in radical hysterectomy type III. Gynecol Oncol 2000; 79:154-7.

11. Kadar N, Saliba N, Nelson JH. The frequency, causes and prevention of severe urinary dysfunction after radical hysterectomy. Br J Obstet Gynecol 1983; 90:858-63.

12. Ralph G, Winter R, Michelistch L, Tomussino K. Radicality of the parametrial resection and dysfunction of the lower urinary tract after radical hysterectomy. Eur J Gynecl Oncol 1991; 12:27_30. 
13. Glahn BE. The neurogenic factors in vesical dysfunction following RH for carcinoma of the cervix. Scand J Urol Nephrol 1970; 4:107-16.

14. Scotti R, Bergman A, Bhatia NN, Ostergard DR. Urodynamic changes in urethrovesical function after radical hysterectomy. Ostet Gynecol 1986; 68:111-120.

15. Seski JC, Diokno AC. Bladder dysfunction after radical hysterectomy. Am J Obstet Gynecol 1977; 128:643-51.

16. Forney JP.The effect of radical hysterectomy on bladder physiology, Am J Obstet Gynecol 2980; 138:374-82

17. Ralph G, Tamussino K, Lichtenegger W. Urological complication after radical abdominal hysterectomy for cervical cancer.Bailliers Clin Obstet Gynecol 1988;2:943-52.

18. Sasaki H, Yoshida T, Noda K, Yachiku S, Minami K, Kaneko S. urethral pressure profiles following radical hysterectomy. Obstet Gynecol 1982; 59: 101-4

19. Possover M, Stober S, Plaul K, and Schneider A. Identification and preservation of the motoric-innervation of the bladder in radical hysterectomy.

20. Mass CP, Kenter GG,De Ruiter MC and Trimbos JB. The autonomic nerves in radical hysterectomy. Inter J Gynecol Cancer, 1999 ; 9:144-9.

21. Sato T. The vascular and neuronal composition of the lateral ligament of the rectum and the rectosacral fascia. Sug Radiol Anat 1991; 13:17-22.

22. Fraser AC. The late effects of Wertheims hysterectomy on the urinary tract.Br J Obstet Gynaecol 1966; 73:1002-1007.

23. Smith PH, Turnbull GA, Currie DW, Peel KR. The urological complications of Wertheims hysterectomy. Br J Urol 1969; 41:685-688. 